\title{
Analytical Model of Non-Imaging Planar Concentrator for the Application in Dense-Array Concentrator Photovoltaic System
}

\author{
Chee-Woon Wong ${ }^{*}$, Kok-Keong Chong and Tiong-Keat Yew \\ Faculty of Engineering and Science, Universiti Tunku Abdul Rahman, Malaysia
}

\begin{abstract}
Instead of using numerical simulation method that is relatively slow if accuracy is required, an analytical model has been proposed to analyze the optical characteristic of Non-Imaging Planar Concentrator (NIPC) for the application in dense-array concentrator photovoltaic (DACPV) system. Several trigonometry equations have been solved to determine the maximum solar concentration ratio, uniform illumination area and energy within the uniform area by varying the focal distance. The concentration ratio can range from 337 to 422 sun sand the energy percentage decreases from $68 \%$ to $50 \%$ within the area that ranges from $82 \mathrm{~cm}^{2}$ to $52 \mathrm{~cm}^{2}$. According to the results, the analytical model is capable of obtaining the NIPC's three important design criteria within an error of at most $4.7 \%$ compare with the numerical simulation method. As a conclusion, analytical model is capable of offering an express method to designer for optimizing the performance of the DACPV system.
\end{abstract}

Keywords: Analytical model, solar concentrator, concentrator photovoltaic, uniform illumination area

\section{Introduction}

Multi-junction solar cells have the potential of achieving conversion efficiency more than $40 \%$ by having three or more materials that are all sensitive to different part of the solar spectrum [1]. In fact, majority of the multi-junction solar cell characterizations have a discrepancy between the provided testing conditions and the real operational conditions. For example, the given cell efficiency is measured under AM1.5D, uniform illumination with high solar concentration ratio and at a temperature of $25{ }^{\circ} \mathrm{C}$. It is an arduous work to fulfill these conditions in the real operation of CPV module that consists of multi-junction solar cells. Several real operation factors are limiting the performance of solar cells, especially the non-uniform illumination that caused current mismatched in the dense-array connection. When non-uniform illumination is directed on the solar cells, there will be a drop in efficiency as compared to the cells operated under uniform illumination [2-4]. In order to achieve high electrical conversion efficiency, solar concentrators that use relatively inexpensive optics such as mirrors or lenses have been developed to deliver uniform flux distribution on the multi-junction solar cells at hundreds to thousands of solar concentration ratio. This approach is capable of reducing the expensive solar cell area by increasing the solar concentration ratio and the light intensity. Over the past few decades, some researchers have put a lot of effort to design various types of non-imaging solar concentrators for producing uniform solar illumination. For example, Mills et al. has proposed a linear Fresnel reflector to produce better uniformity of solar irradiation on the receiver [5]. However, the solar concentration ratio of the proposed solar concentrator is less than 100 suns.

In year 2006, Ryu et al has introduced modular Fresnel concentrator for achieving moderate solar concentration ratio of up to 121 suns and better uniform illumination, but the reflection at the lens surface and absorption by the lens material have caused the transmission efficiency of less than $80 \%$ [6]. In year 2009, Chong at el. has presented the design and construction of Non-Imaging Planar Concentrator (NIPC) for the application in densearray concentrator photovoltaic (DACPV) system [7].

The NIPC is capable of producing much more uniform spatial irradiance and reasonable high solar concentration ratio for maintaining a high output power and stability of the DACPV system that consists of bypass diodes and multi-junction solar cells connected in series and parallel. On the other hand, a comprehensive characteristic analysis of solar flux distribution on the receiver plane of the NIPC

*Corresponding author: Address: Faculty of Engineering and Science, Universiti Tunku Abdul Rahman, Jalan Genting Kelang, Setapak 53300 Kuala Lumpur, Malaysia E-mail address: wcwoon@utar.edu.my, Phone: +60341079802 Fax: +60341079803 
has been carried out by the use of numerical simulation method [8]. The numerical simulation represents all of the NIPC behaviors, but this method is relatively slow in terms of computer time if accuracy is required.

For the sake of shortening the simulation time, an analytical model has been proposed in this paper in order to analyze the optical characteristic of NIPC. By changing of the $f / D$ ratio, the maximum solar concentration ratio and uniform illumination area of NIPC have been determined by several trigonometry equations.

\section{Analytical Model of NIPC}

Instead of using a single piece of parabolic dish, the NIPC comprising of multi-faceted mirrors acts as the optical aperture to collect and to focus the incident sunlight at any focal distance along the optical axis (see Figure 1). Solar disk effect is considered in the optical design and analysis, where the sunlight that strikes on the mirror surface and the receiver are treated as cone rays.

$$
\begin{aligned}
& \theta=\frac{1}{2} \arctan \left[\frac{\sqrt{H_{C x}^{2}+H_{C y}^{2}}}{f}\right] \\
& \gamma=\arctan \left[\frac{H_{C y}}{f+\sqrt{H_{C x}^{2}+H_{C y}^{2}+f^{2}}}\right]
\end{aligned}
$$

$$
\sigma=\arctan \left[\frac{H_{C x}}{\left(H_{C x}^{2}+H_{C y}^{2}+2 f^{2}+2 f \sqrt{H_{C x}^{2}+H_{C y}^{2}+f^{2}}\right)^{1 / 2}}\right]
$$

For a geometrical representation, a general Cartesian coordinate system is defined in the plane of the solar concentrator, with its origin located at the center of the concentrator, $x$-axis lying along the row of mirrors, $y$ axis lying along the column of mirrors and $z$-axis pointing towards the receiver $\left(T_{x}, T_{y}, T_{z}\right)$. The position for each mirror in the planar concentrator can be indexed as $(i, j)$, where $i$ and $j$ express the mirror located at $i$-th row and $j$-th column of the concentrator.

A sub-coordinate system $\left(x^{\prime}, y^{\prime}, z^{\prime}\right)$ is defined at the local facet mirror with the origin located at the center of mirror. Referring to Figure 2, $\left(H_{C x}, H_{C y}, H_{C z}\right)_{i j}$ represents the coordinate of the central point (or pivot point) for an $i, j$-mirror and the coordinate of the focal point for the concentrator is $(0,0, f) . \hat{I}, \hat{N}$ and $\hat{R}$ are the unit vectors of the incident ray, the normal of $i, j$ mirror and the reflected ray respectively. The incident angle $(\theta)$ of the sunray, relative to $i, j$-mirror, and the tilted angles of $i, j$-mirror about $x^{\prime}$-axis $(\gamma)$ and $y^{\prime}$-axis $(\sigma)$ can be determined by using Snell-Descartes law.

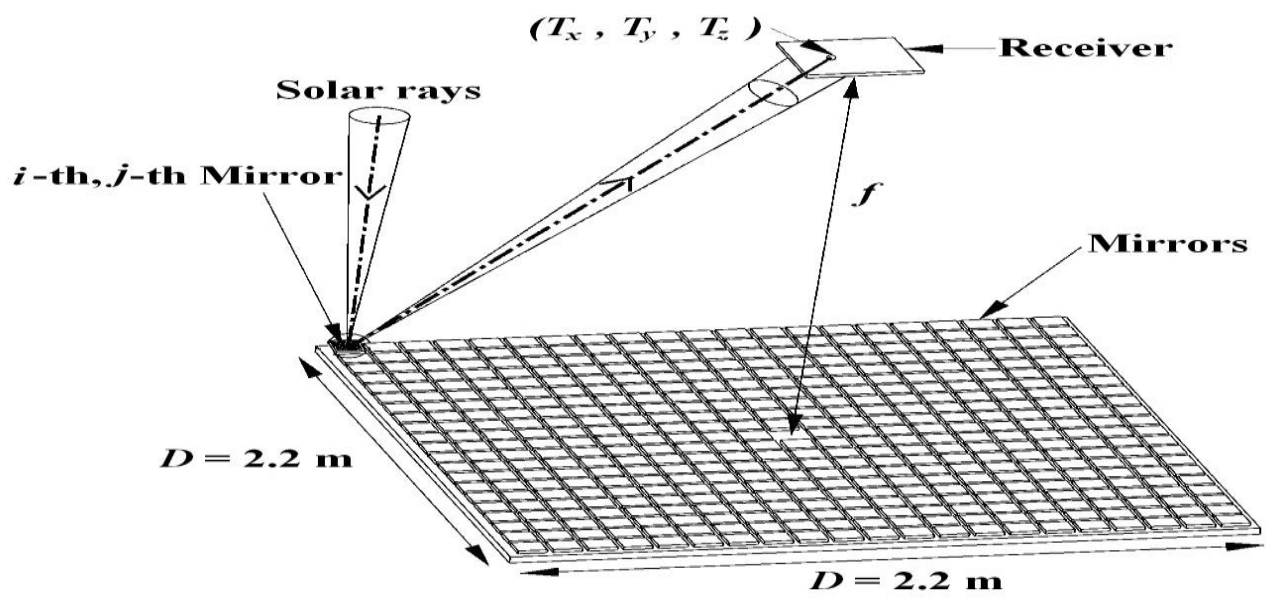

Figure 1. Conceptual layout design of the Non-Imaging Planar Concentrator (NIPC). 
Cross-section view of the planar concentrator shows how the individual mirror with a dimension $\mathrm{W} \times \mathrm{W}$ directs the sunlight to the receiver is illustrated in Figure 3. It can be seen that the rate of solar energy falling on both receiver surfaces is the same. However, the area of receiver surface $\mathrm{A}$ is greater than its projection; hypothetical receiver surface B which is normal to the reflected solar rays. Because of the cosine effect, the rate of solar energy per unit area that falling on receiver surface $\mathrm{A}$ is less than on receiver surface B. Assume that the solar energy falling on the hypothetical receiver surface B is considered as 1 sun. Therefore, the total solar concentration ratio, $\mathrm{C}$ after superposing all the mirror images on the receiver surface $\mathrm{A}$ is

$$
C=\sum \cos 2 \theta_{i j}
$$

where the angle $\theta_{i j}$ is the incident angle of solar rays on the $i, j$-mirror.

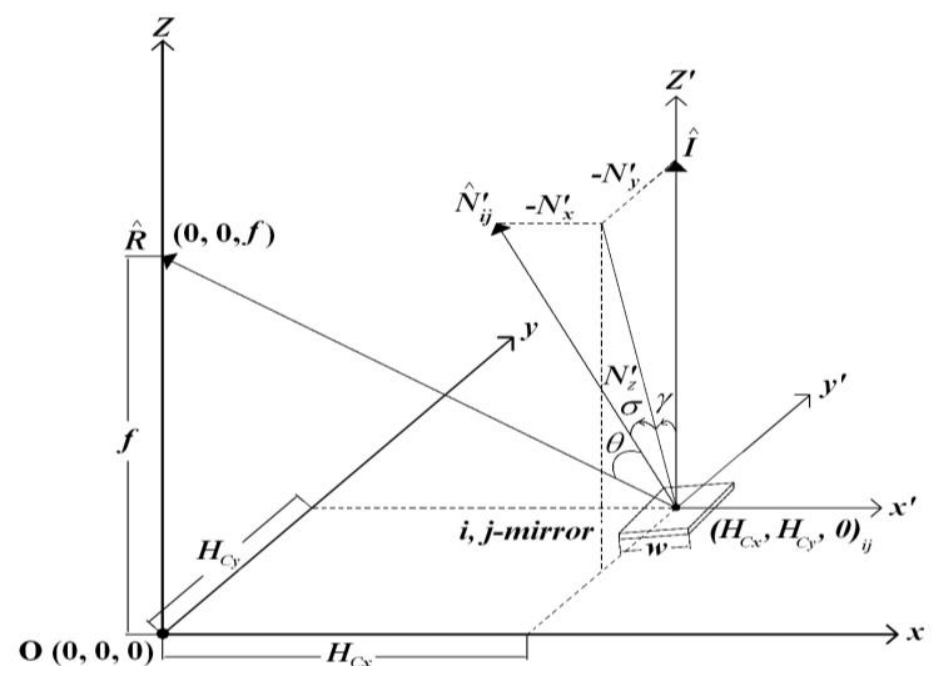

Figure 2. Cartesian coordinate system used to represent the main coordinate system $(x, y, z)$ at the plane of the planar concentrator and the sub-coordinate system $\left(x^{\prime}, y^{\prime}, z^{\prime}\right)$ is defined at the local facet mirror.

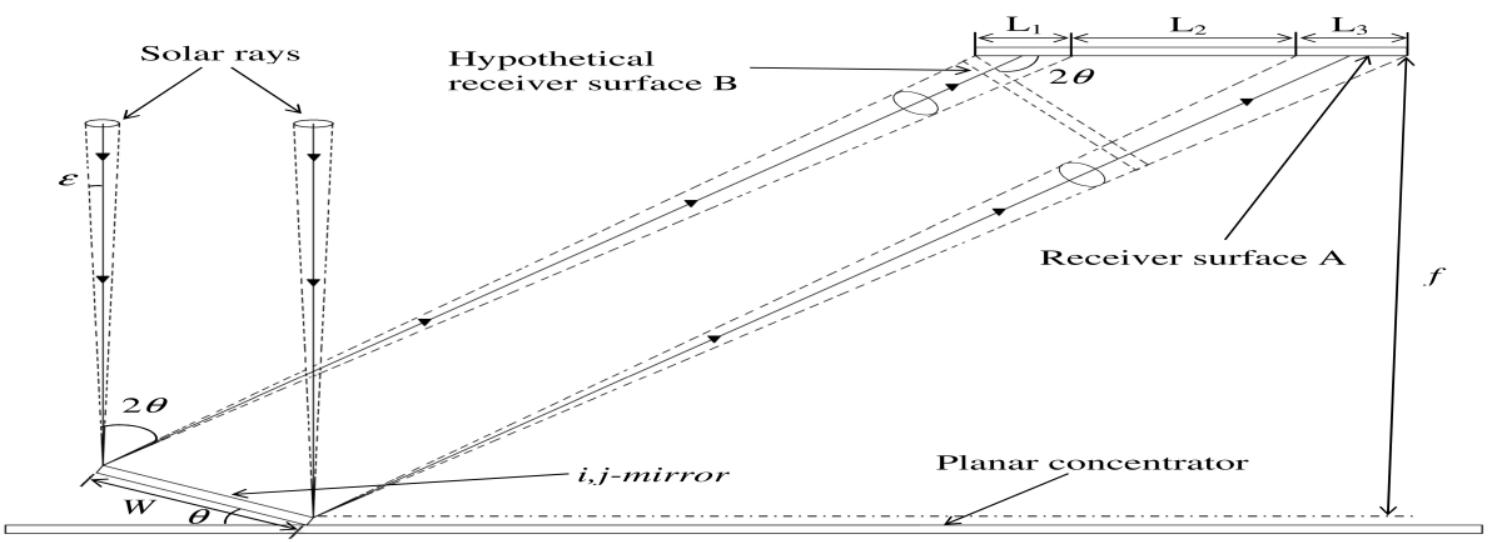

Figure 3. Cross-section view of the planar concentrator shows how the individual mirror with a dimension $\mathrm{W} \times \mathrm{W}$ directs the sunlight to the receiver.

By using the trigonometry theorem and considering the solar disk half angle $(\varepsilon)$ of $4.65 \mathrm{mrad}$, the size of the solar image that cast on the receiver surface A can be determined as follow: 
$L=L_{1}+L_{2}+L_{3}=f[\tan (2 \theta+\varepsilon)-\tan (2 \theta-\varepsilon)]+$

$W[\cos \theta+\sin \theta \tan (2 \theta-\varepsilon)]$

where

$$
\begin{aligned}
& L_{1}=(f-W \sin \theta)[\tan (2 \theta+\varepsilon)-\tan (2 \theta-\varepsilon)] \\
& L_{2}=W \cos \theta+f \tan (2 \theta-\varepsilon)- \\
& (f-W \sin \theta) \tan (2 \theta+\varepsilon) \\
& L_{3}=f[\tan (2 \theta+\varepsilon)-\tan (2 \theta-\varepsilon)]
\end{aligned}
$$

Since the idea of NIPC is superposing all the mirror images into one, the overall solar image area, $A$ and the uniform illumination area, $A_{\text {uniform }}$ on the receiver A can be determined from the Equation 5 and Equation 7 respectively.

$$
\begin{aligned}
& A=\left\{\begin{array}{l}
f\left[\tan \left(2 \theta_{\text {max }}+\varepsilon\right)-\tan \left(2 \theta_{\text {max }}-\varepsilon\right)\right] \\
+W\left[\cos \theta_{\text {max }}+\sin \theta_{\text {max }} \tan \left(2 \theta_{\text {max }}-\varepsilon\right)\right]
\end{array}\right\}^{2} \\
& A_{\text {un f̊ } r m}=\left\{\begin{array}{l}
W \cos \theta_{\text {min }}+f \tan \left(2 \theta_{\text {min }}-\varepsilon\right) \\
-\left(f-W \sin \theta_{\text {min }}\right) \tan \left(2 \theta_{\text {min }}+\varepsilon\right)
\end{array}\right\}^{2}
\end{aligned}
$$

Besides that, the percentage of energy in uniform illumination area is a measurement of energy distributed in the uniform illumination area compared with the total input energy, and it is calculated as

$$
E=\frac{C \times A_{\text {unĭo } r m}}{W^{2} \sum \cos \theta_{i j}}
$$

\section{Results and Discussion}

In this paper, an optical analysis of the planar concentrator, composed of $21 \times 21$ facet mirrors with a dimension of $10 \mathrm{~cm} \times 10 \mathrm{~cm}$ each to form a total reflective area of $4.4 \mathrm{~m}^{2}$ and a width of $2.2 \mathrm{~m}$, has been carried out using the aforementioned methodology by changing of the $f I D$ ratio, where $f$ and $D$ are the focal distance and the width of the planar concentrator correspondingly. To quantify the performance of NIPC that is specially designed to be incorporated in DACPV system, three different performance studies including the average solar concentration ratio in uniform area, the uniformity of solar illumination and the energy in uniform illumination area have been conducted for different $f / D$ ratio from 0.4545 to 1.3635 with the increment of 0.04545 each time.

Figure 4 has been plotted to reveal the uniform illumination area varies in the range from $82 \mathrm{~cm}^{2}$ to 52 $\mathrm{cm}^{2}$ for different $f / D$ ratio. According to the result, the higher the focal distance $(f)$ the lower the uniform illumination area will be formed on the receiver plane. Depending on the $f / D$ ratio (from 0.4545 to 1.3635 ), the maximum solar concentration ratio and the percentage of energy in the uniform illumination area has been illustrated in Figure 5 and Figure 6 respectively. With the change of the focal distance, the maximum solar concentration ratio and percentage of energy in uniform illumination area vary in the range from 336 to 422 suns and from $68 \%$ to $52 \%$ correspondingly.

From the simulated results, we can conclude that the maximum solar concentration ratio improves when the focal distance increases but at the same time the percentage of energy in uniform illumination area will be sacrificed. For that reason, a trade-off between solar concentration ratio and total energy in uniform illumination area has to be sought to obtain the best performance of the concentrator. Furthermore, the comparison between analytical model and numerical simulation data are also shown in Figure 4, Figure 5 and Figure 6. It shows that analytical model has an error of at most $4.7 \%$ to determine all the important design criteria compare with numerical simulation method. 


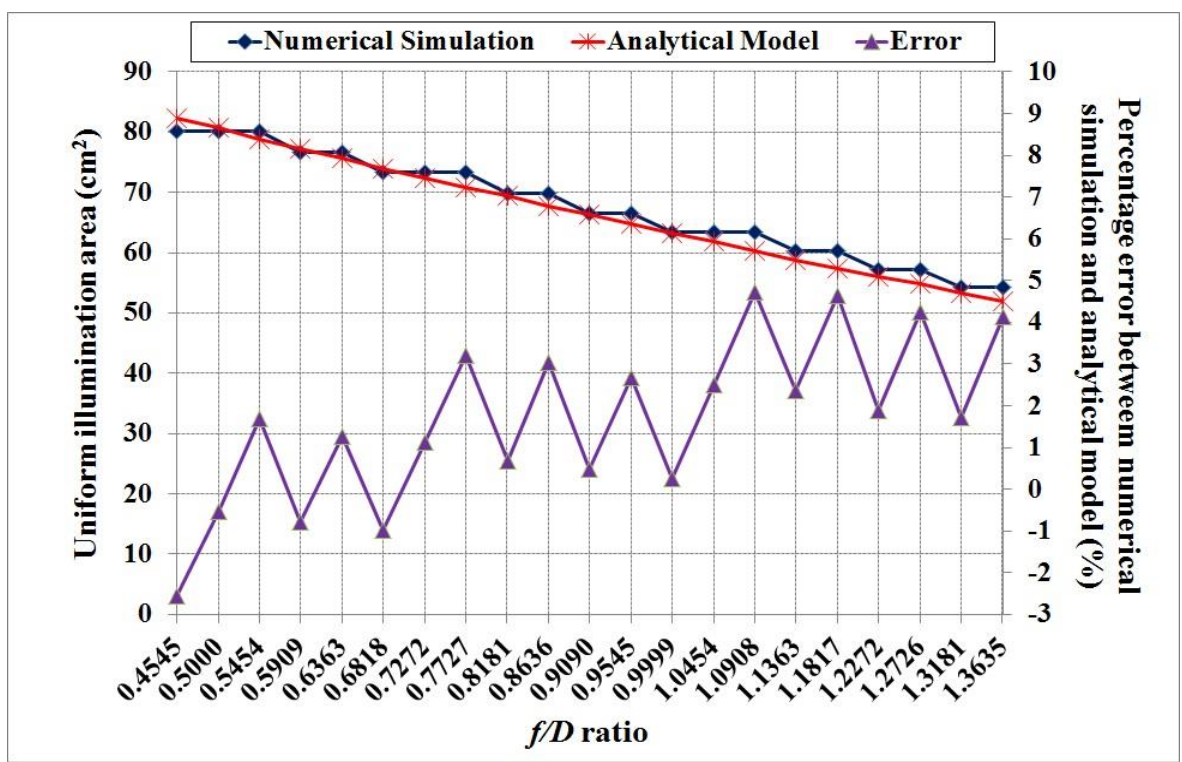

Figure 4. Graph shows both the uniform illumination area and the percentage error between numerical simulation and analytical model versus $f / D$ ratio.

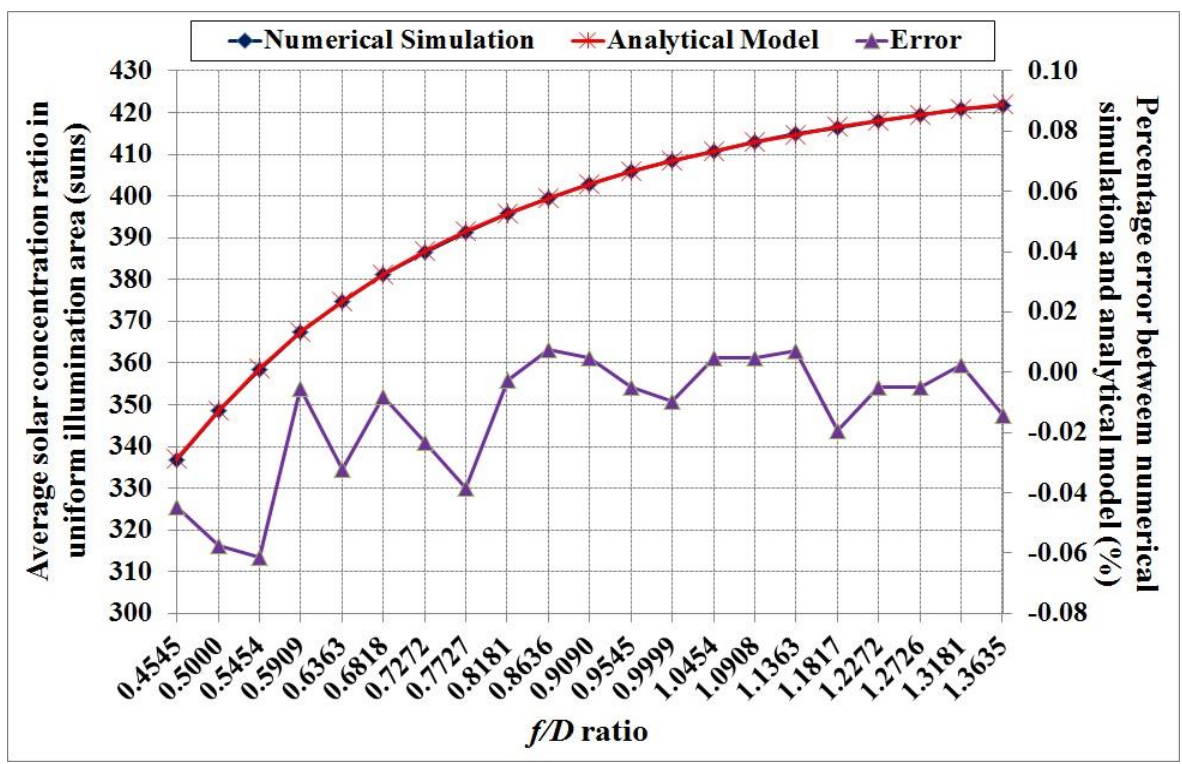

Figure 5. Graph shows both the average solar concentration ration in uniform illumination area and the percentage error between numerical simulation and analytical model versus $f / D$ ratio. 


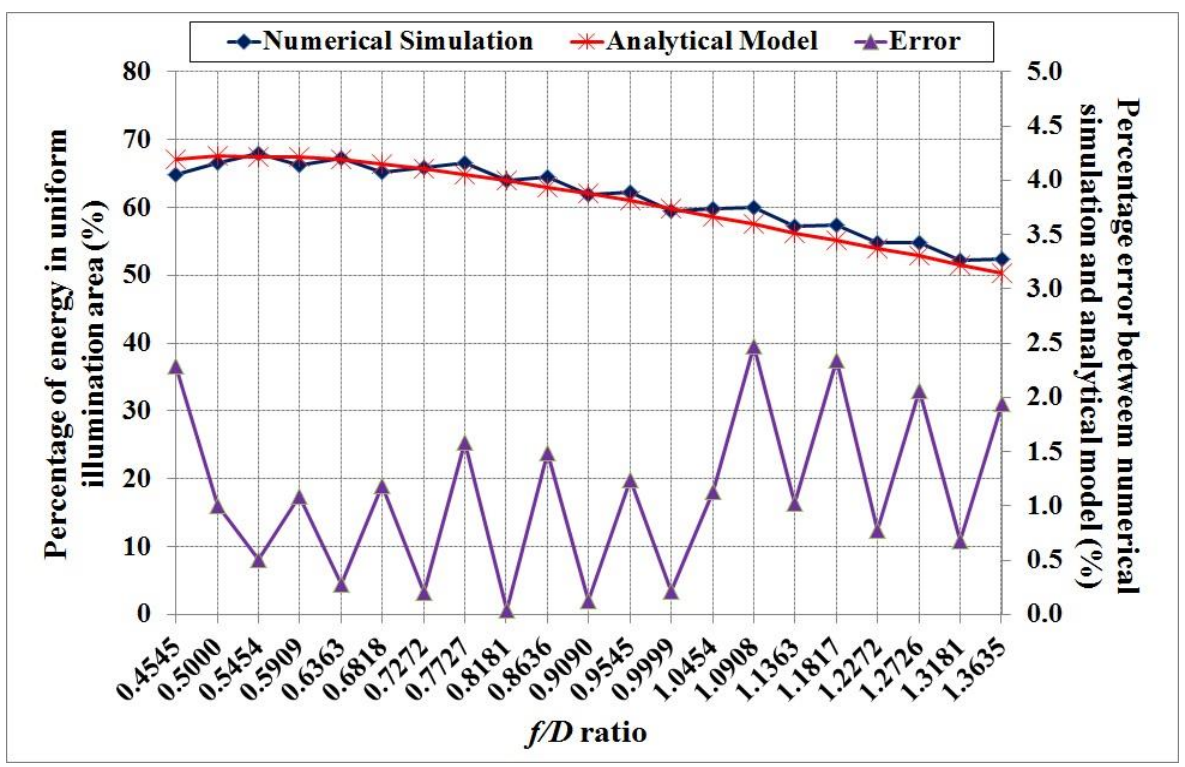

Figure 6. Graph shows both the percentage of energy in uniform illumination area and the percentage error between numerical simulation and analytical model versus $f / D$ ratio.

\section{Conclusions}

The principle and the methodology of analytical model for analyzing the optical characteristic of the NonImaging Planar Concentrator have been described in detail. According to the simulation results, the designer has to seek a trade-off between solar concentration ratio and total energy in uniform illumination area in order to obtain the best performance for the CPV system. Since the maximum solar concentration ratio increases with the focal distance but at the same time the percentage of energy in uniform illumination area will be surrendered. In addition, the results show that analytical model is capable of obtaining the solar concentration ratio, the uniform illumination area and the total energy in uniform illumination area within an error of at most $4.7 \%$ compare with the numerical simulation method. As a conclusion, analytical model is capable of offering an express method to designer for optimizing the performance of the DACPV system with uniform flux distribution and reasonable high solar concentration ratio.

\section{Acknowledgements}

The authors would like to express their gratitude to Fundamental Research Grant Scheme (FRGS) with project number FRGS/1/11/TK/UTAR/03/4 which supported by the Ministry of Higher Education Malaysia.

\section{References}

[1] King RR, BocaA, HongW, Law D, Kinsey G, Fetzer C, Haddad M, Edmondson K, Yoon H, Pien P, Karam N. High-Efficiency Multijunction Photovoltaics for Low-Cost Solar Electricity. 21st Annual Meeting of The IEEE Lasers \& ElectroOptics Society 2008.

[2] Andreev V, Grilikhes V, Rumyantsev V, Timoshina N, Shvarts M. Effect of Nonuniform Light Intensity Distribution on Temperature Coefficients of Concentrators Solar Cells. 3rd World Conference on Photovoltaic Energy Conversion 2003.

[3] Nishioka K, Takamoto T, Agui T, Kaneiwa M, Uraoka Y, Fuyuki T.Evaluation of InGaP/InGaAs/Ge Triple-junction Solar Cell and Optimization of Solar Cell's Structure Focusing on Series Resistance for High-Efficiency Concentrator Photovoltaic Systems. Solar Energy Materials \& Solar Cells 2006; 90: 13081321.

[4] Verlinden PJ, Lewandowski A, Bingham C, Kinsey GS, Sherif RA,Lasich JB.Performance and Reliability of Multijunction III-V Modules for Concentrator Dish and Central Receiver Applications. Conference Record of the IEEE 4th World Conference on Photovoltaic Energy Conversion 2006. [5] Mills DR,Morrison GL.Compact Linear Fresnel Reflector Solar Thermal Powerplants. Solar Energy 2000; 68: 263-283.

[6] Ryu K, Rhee JG, Park KM, Kim J.Concept and Design of Modular Fresnel Lenses for Concentration Solar PV System. Solar Energy 2006; 80: 1580-1587. 
[7] Chong KK, Siaw FL, Wong CW, Wong GS. Design and Construction of Non-Imaging Planar Concentrator for Concentrator Photovoltaic System. Renewable Energy 2009; 34: 1364-1370.
[8] Chong KK, Wong CW, Siaw FL,Yew TK. Optical Characterization of Non-Imaging Planar Concentrator for the Application in Concentrator Photovoltaic System. Journal of Solar Energy Engineering 2010; 132: 011011-011019. 